\title{
Investigations in Respect to the Determination of Cavitation Intensity by the Means of Acoustic Signals
}

\author{
Recherches sur la détermination de l'intensité de cavitation \\ d'après des signaux acoustiques
}

\author{
Bernd Stoffel, Prof. Dr.-Ing. \\ Werner Schuller, Dipl.-Ing. \\ Technical University of Darmstadt \\ Institute for Hydraulic Machines and Systems \\ Magdalenenstr. 4, D 6100 Darmstadt, Germany \\ Tel. : (06151) 162153; Telefax : (06151) 162453
}

\section{Introduction}

For the limitation of cavitation in respect to the avoidance or limitation of erosion a method for the determination and quantification of the hydrodynamic cavitation intensity $(\mathrm{HCI})$ is needed. Investigations to find correlations between erosive intensity and noise emission of cavitation were reported for simple test configurations in [2], [3] and [4] and for hydraulic turbomachines in [1], [5] and [6]. If such a correlation can be confirmed, acoustic sensors could be used for the indirect determination of the HCI. In order to gain quantitative information from the acoustic signals, on-site calibration of the measuring chains seems to be an absolutely necessary prerequisite.

\section{Test facility and test configurations}

The test section with a rectangular cross section was installed in a closed loop equipped with all devices which are necessary for the control and measurement of the upstream velocity $v_{0}$, the upstream pressure $p_{0}$, the temperature and the gas content of the water. Part of the walls of the test section were made of Plexiglass to enable the visual observation of the cavitation patterns. Miniature hydrophones (BRÜEL \& KJAER, Type 8103), pressure transducers (KISTLER, Type $601 \mathrm{~A}$ ) and Acoustic Emission (AE) transducers (BRÜEL \& KJAER, Type 8312) were used as sensors.

The test configurations (see Fig. I) are based on a common concept which consists in the abstraction of the flow field and cavitation patterns from the real situation in pump impellers to simpler two-dimensional configurations. The first step of abstraction leads to a single hydrofoil showing a good geometrical similarity to an impeller blade. The second step leads to a Venturi-section of varying rectangular cross section where only the velocity, the pressure distribution and the cavitation pattern show similarity to those found in pump impellers but the geometry is more suitable for the insertion of probes and specimens.

The hydrofoil has a NACA 0015 cross section which was somewhat modified by increasing the nose radius to $6 \%$ of the chord length (see Fig. 2). The hydrofoil was fixed at mid-height of the test section at different angles of attack $\delta$. The Venturi-contour is characterized by the fact that an essential part of the curved boundaries is formed by a cylinder which is partly immersed into the test section.

\section{Recherches sur la détermination de l'intensité de cavitation d'après des signaux acoustiques}

Les signaux acoustiques reçu par un détecteur placé en dehors d'une zone de cavitation sont utilisés pour la détermination de l'intensité des implosions de bulles. La méthode présentée est basée sur l'application des fonctions de transfert déterminées expérimentalement qui prennent en considération la transmission des ondes de pression et leur conversion en signaux électriques. Les résultats obtenus se réfèrent à la cavitation sur un profil et dans un canal de Venturi. 


\section{Hydrofoil}

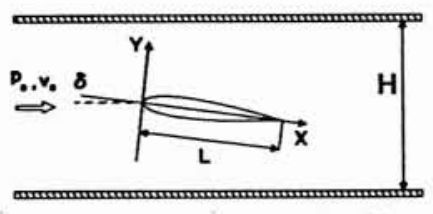

L. $100 \mathrm{~mm}$

\section{Venturi}

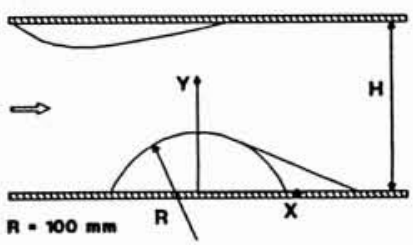

1. Test configurations.

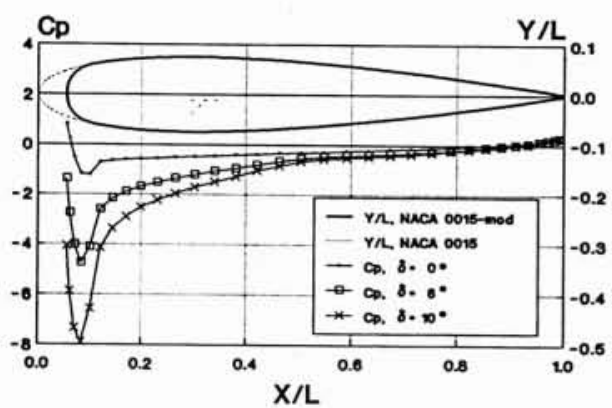

2. Geometry and theoretical pressure distribution (suction side) of the hydrofoil.

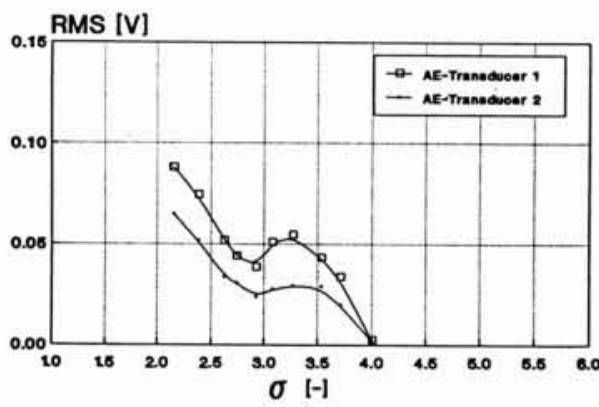

4. Acoustic signals versus cavitation number. Hydrofoil. $\left(v_{0}=\right.$ $8 \mathrm{~m} / \mathrm{s}, \delta=6^{\circ}$, AE-Transducer at different locations).

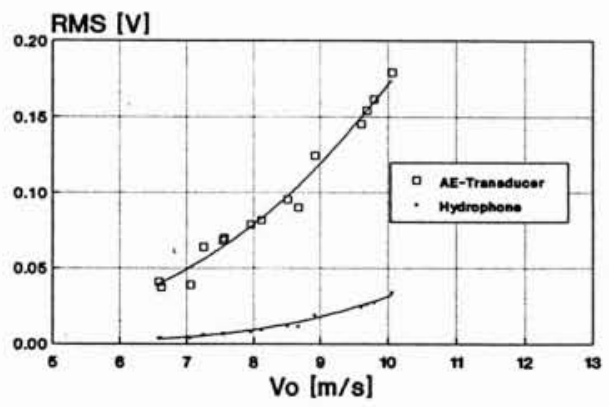

6. Influence of velocity on acoustic signals. Hydrofoil, $\sigma=2.75$.

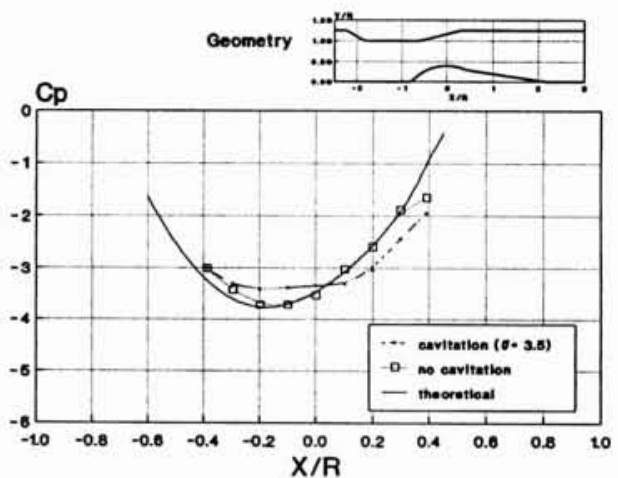

3. Geometry and pressure distribution of the Venturi. Comparison of calculation with measurements under cavitating and noncavitating conditions.

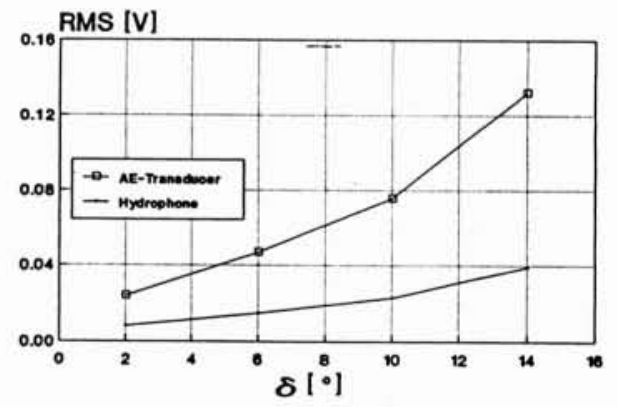

5. Influence of angle of attack on acoustic signals at constant velocity $\left(v_{0}=8 \mathrm{~m} / \mathrm{s}\right)$ and cavitation length $(L c / L=0.2)$.

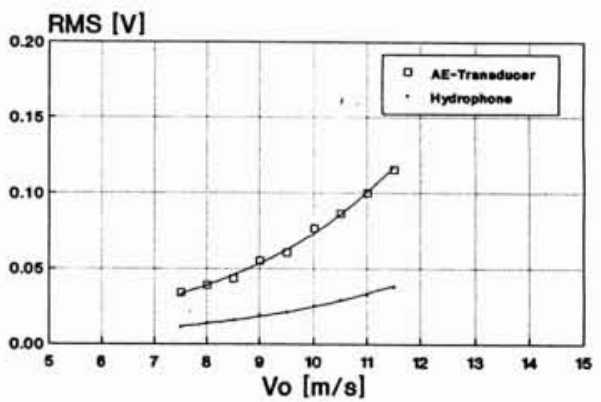

7. Influence of velocity on acoustic signals. Venturi, $\sigma=3.4$. original signals

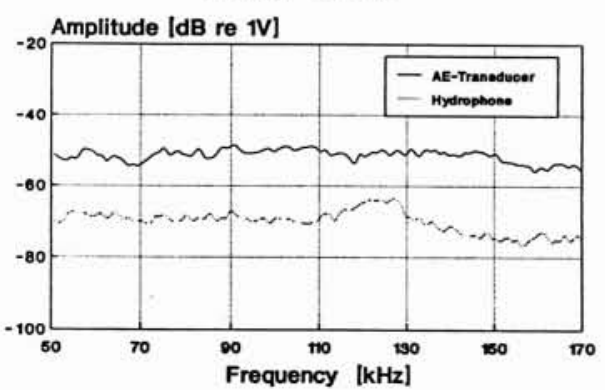

after application of TF

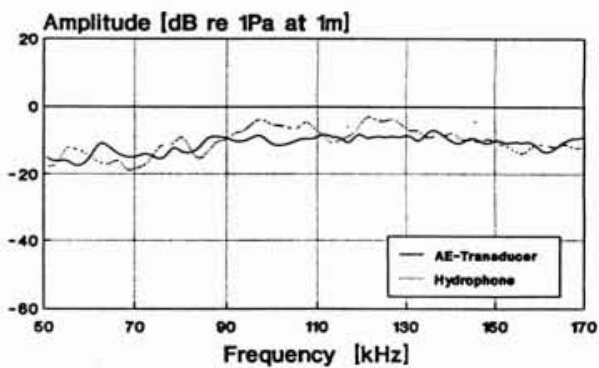




\section{Pressure distribution and cavitation patterns}

The theoretical pressure distribution was calculated using the method of singularities and assuming a twodimensional inviscid flow. Fig. 2 shows some pressure distributions along the low pressure surface (suction side) of the hydrofoil for various angles of attack $\delta$. The dimensionless pressure coefficient $c_{p}$ is defined in the usual way as the pressure difference $\left(p-p_{0}\right)$ divided by the dynamic pressure of the velocity $v_{0}$. For the Venturi configuration the calculated pressure distribution is compared with experimental data in Fig. 3 and shows a good agreement for non cavitating conditions. The measured pressure distribution for the case of developed cavitation $(\sigma=3.5$ at $v_{0}=8 \mathrm{~m} / \mathrm{s}$ ) shows the well known limitation of $c_{p, \min }$ to the value of $-\sigma$ and the downstream shift of pressure increase caused by the vapour filled cavities.

In respect to the development of cavitation patterns with decreasing $\sigma$, a similar behaviour was observed for the Venturi and for the hydrofoil. The cavitation starts in the corners and with individual strips which merge and grow downstream until transition to detached and cyclic cavitation and further increase of the (time-averaged) cavitation length take place.

\section{Dependence of acoustic signals on cavitation characteristics}

As a typical result, with varying length or changing type of cavitation the frequency spectrum of signals only more or less shifts parallely (in the direction of the amplitude axis) without significant modification of the shape of the curves. This means that the final electrical signal are much more characterized by the transmission and conversion of the pressure waves and the subsequent signal conditioning than by the source of the acoustic signals itself. Based on these findings and on the assumption that the effects of transmission and conversion are linear, one may expect that variations of the strength of the acoustic source must lead to corresponding proportional variations of global quantities of the final signals (as e.g., the rms-value).

Fig. 4 shows the variation of the rms-value with the cavitation number for the test configuration with the hydrofoil $\left(\delta=6^{\circ}, v_{0}=8 \mathrm{~m} / \mathrm{s}, \mathrm{AE}\right.$ transducer at different locations). The similar shape of the curves illustrates the strong correlation between the rms-values of the simultaneously registered acoustic signals of the two transducers. For both configurations, the same general dependence of acoustic signals on cavitation number exists, which is furtheron strongly correlated with variations and changes in cavitation patterns. With a decreasing cavitation number $\sigma$, after inception of cavitation the rms-values increase, pass through a local maximum and drop down to a local minimum when the cavitation begins to cover the whole span as a closed sheet, then increase again with further growth of the cavitation length combined with the onset of detaching and cyclic cavitation.
The configuration with the hydrofoil made possible investigations on the effect of the pressure gradient by varying the angle of attack $\sigma$ at constant velocity $v_{0}$ and constant cavitation length $L c$. The pressure gradient is increasing with the angle of attack and this leads to an corresponding increase of the rms-values of the acoustic signals (see Fig. 5).

The dependence of the rms-values on the velocity $v_{0}$ at constant cavitation number $\sigma$ is presented in Figs. 6 and 7 for the two configurations, respectively. The curves can be well approximated by a proportionality between the rms-value and $v_{0}{ }^{n}$ with a velocity exponent $n$ from 3 to 4 . Assuming the pressure gradient proportional to $v_{0}{ }^{0}$, the total number of nuclei supplied to the cavitation zone as proportional to $v_{0}$ and the number of nuclei becoming critical as increasing more with the velocity than the total number of nuclei, an exponent $\mathrm{n}>3$ seems to be realistic. It also corresponds to the velocity exponents of 6 to 8 which are usually reported for cavitation erosion taking into account that the intensity of bubble implosions is related to the square of the implosion pressures.

\section{On site calibration and application of transfer func- tions}

Based on the facts reported above, it seems possible to evaluate the absolute strength of bubble implosion energy if the transfer function between the pressure waves and the final electrical signal is known. This transfer function, including the effects of pressure wave attenuation in the liquid, reflection and absorption at the walls, transducer and filter response can be determined experimentally using an acoustic source with a known transmitting characteristic placed temporarily at the location where the cavitation bubble implosions are expected (« on-site calibration "). In the frame of our investigations, two different sources were tested: a miniature hydrophone used as transmitter and a device for generating sparc induced single bubbles. The transmitting characteristics of the two sources were calibrated in advance by submerging them in a large water reservoir of our laboratory. Hydrophones with known receiving characteristics served to determine the transmitted pressure amplitudes assuming free-field conditions for the transmission of pressure waves.

Fig. 8 shows an example of the application of transfer functions to cavitation related signals. The upper diagram shows the frequency spectra of the original voltage signals which were received and converted simultaneously by a hydrophone located upstream of the test section and by an AE transducer attached to the outer surface of the test section. In the lower diagram, the results of dividing the original frequency spectra by the corresponding transfer functions are plotted as pressure amplitudes referred to free-field conditions at a distance of $1 \mathrm{~m}$. In spite of the very different voltage output of the transducers, a good agreement results in respect to the pressure amplitudes calculated therefrom by the application of transferfunctions. This method, therefore, seems very promising for the acoustical determination of HCI. 


\section{References}

[1] GÖLICH F. - " Beitrag zur Bestimmung der Kavitationserosion in Kreiselpumpen auf Grund der Blasenfeldlänge und des Kavitationsschalls ». Doct. Thesis, TH Darmstadt, 1989.

[2] Lush P.A. - «Correlation of Cavitation Erosion Rate and Sound Pressure Level ». Int. Symp. Cav. Noise, ASME, Phoenix, 1982.

[3] Ramamurthy A.S. - "Velocity Exponent for Erosion and Noise Due to Cavitation». Joint ASME, Appl. Mech., Fluids Engng. + Bioengng. Conf., Niagara Falls, 1979.

[4] OKaвE Y. et al. - « Experimental Studies on Relationship Between Erosion Rate and Apparent Impact Pressure and Cavitation Monitoring System by Acoustic Detector ". Int . Symp. Cav., Sendai, 1986.

[5] KIREJZYK J.- « Über die Beurteilung der Kavitationsintensität in Pumpen ». Pump Conf., Karlsruhe, 1984.

[6] Steller K. - « Prediction of Cavitation Damage in Hydraulic Turbomachinery ». 7th Conf. Fluid Mach., Budapest, 1983. 